\title{
Preservación de la fertilidad en una paciente con teratoma bilateral. Reporte de un caso y revisión de la literatura
}

\author{
Preservation of fertility in a patient with bilateral teratoma. Case report and literature review
}

\author{
Henry A. Mateo-Sánez ${ }^{1 *}$, Daniela Mateo-Madriga, Adrián Dávalos-Álvarez³, \\ Fernanda Domínguez-Dorame ${ }^{4}$ y Jeanneth Ku-González ${ }^{4}$ \\ 'Departamento de Ginecología y Obstetricia, Hospital Santa Rosa de Lima, Ensenada, Baja California; ${ }^{2}$ Servicio de Genética Médica, Hospital \\ Infantil de México, Ciudad de México; ${ }^{3}$ Departamento de Ginecología en IVF Fertility Clinic, Guadalajara, Jalisco; ${ }^{4}$ Servicio de Ginecología y \\ Obstetricia, Hospital Santa Rosa de Lima, Ensenada, Baja California. México
}

\begin{abstract}
Resumen
Los tumores de células germinales son una variante de los tumores ováricos, siendo el teratoma el más frecuente. Del total de los tumores ováricos diagnosticados, el 12\% se encontrarán en pacientes en edad fértil. Se presenta el caso de una paciente de 22 años que acude a consulta con un diagnóstico presuntivo de teratoma bilateral en búsqueda de alternativas terapéuticas, ya que se le planteó originalmente ooforectomía bilateral. Tras un abordaje completo se corroboró el diagnóstico y se procedió a la resección tumoral, logrando la enucleación completa de ambos teratomas y preservando el tejido ovárico, su funcionalidad y su fertilidad.
\end{abstract}

Palabras clave: Preservación de la fertilidad. Teratoma bilateral. Teratoma maduro. Tumor de células germinales.

\begin{abstract}
Germ cell tumors are a variant of ovarian tumors, with teratoma being the most frequent. Of the total number of diagnosed ovarian tumors, $12 \%$ are found in patients of childbearing age. In this case, we present a 22-year-old patient who comes to a consultation with a presumptive diagnosis of bilateral teratoma in search of therapeutic alternatives, since she was originally presented with a bilateral oophorectomy. After a complete approach, the diagnosis was corroborated and tumor resection was carried out, achieving complete enucleation of both teratomas, preserving the ovarian tissue, thus its functionality and fertility.
\end{abstract}

Key words: Preservation of fertility. Bilateral teratoma. Mature teratoma. Germ cell tumor.

\section{Antecedentes}

Los teratomas quísticos benignos se componen de estructuras histológicas maduras de origen ectodérmico, mesodérmico y endodérmico. Generalmente cuentan con un contenido de material sebáceo, células escamosas y cabello, y además pueden contener una variedad importante de otros componentes histológicos, como tejido conectivo, neural, adiposo y óseo, y epitelio respiratorio'. Aunque en el transcurrir de los últimos años han surgido

\section{Correspondencia:}

*Henry A. Mateo-Sánez

Avda. Iturbide, 399

Col. Obrera

Fecha de recepción: 12-05-2020

C.P. 22830, Ensenada, B.C., México

E-mail: hmfertil@hotmail.com

DOI: $10.24875 / C I R U .20000480$
Cir Cir. 2020;88(S2):84-89

Contents available at PubMed

www.cirugiaycirujanos.com (http://creativecommons.org/licenses/by-nc-nd/4.0/) 
varias teorías en torno a su origen, se sigue considerando la partenogénesis como la teoría más aceptada, es decir, que su origen radica exclusivamente en el material genético del ovocito².

Los tumores ováricos son una de las patologías oncoginecológicas más frecuentes, y entre ellos los más frecuentes son los tumores epiteliales de ovario, aunque es bien sabido que en mujeres jóvenes, en edad reproductiva como la paciente de este caso, suelen ser mucho más frecuentes los tumores de células germinales, en particular el teratoma maduro, coloquialmente conocido como quiste dermoide, que abarca hasta el 95\% de esta clase de tumores. En general su comportamiento es benigno, por lo que varios autores han sugerido el manejo expectante de estas pacientes, aunque, por otro lado, el riesgo de torsión y de malignización con necesidad posterior de ooforectomía total son una realidad, por lo que debe plantearse la necesidad de una intervención adecuada y oportuna ${ }^{3,4}$.

\section{Caso clínico}

Mujer de 22 años que acude a la Clínica de Fertilidad del Hospital Santa Rosa de Lima en búsqueda de alternativas terapéuticas por diagnóstico presuntivo de teratoma ovárico bilateral en febrero de 2019, ya que el manejo que se le sugirió consistía en ooforectomía bilateral, a lo cual la paciente se negó.

Como antecedentes heredofamiliares de relevancia, la paciente menciona abuelas paterna y materna con cáncer de mama; madre con diagnóstico de hipotiroidismo; dos hermanas con síndrome de ovario poliquístico, quienes requirieron tratamiento de fertilidad para lograr un embarazo, una de ellas con hipotiroidismo; y prima (por vía materna) diagnosticada con teratoma ovárico derecho a los 24 años, sin especificar la estirpe, manejado con ooforectomía unilateral.

Antecedentes ginecoobstétricos: menarca a los 15 años, con ciclos menstruales de $90 \times 6$, nuligesta. Menciona diagnóstico de síndrome de ovario poliquístico a los 18 años de edad, por lo que recibió tratamiento con valerato de estradiol/dienogest por irregularidad menstrual, usado hasta la fecha de la valoración. No presenta antecedentes quirúrgicos ni alérgicos.

En febrero de 2019 la paciente solicitó atención médica por primera ocasión refiriendo como único síntoma dolor ocasional de tipo punzante en la fosa iliaca izquierda. En el abordaje se llegó al diagnóstico de teratoma ovárico bilateral, por lo que le fue sugerido el manejo con ooforectomía bilateral. Acude por

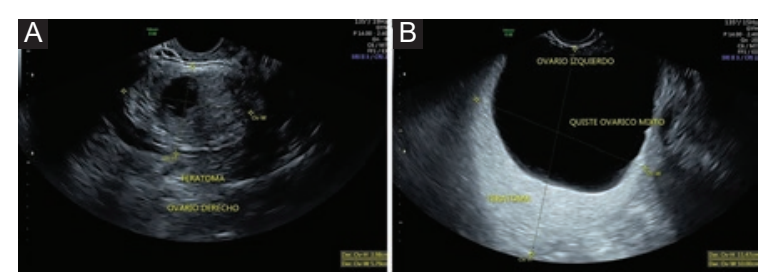

Figura 1. Tumoraciones observadas mediante ultrasonido endovaginal. A: teratoma de ovario derecho. B: teratoma mixto de ovario izquierdo.

primera vez a nuestra consulta en marzo de 2019 solicitando una segunda opinión.

En la exploración física se encuentra: frecuencia cardiaca 80 I.p.m., frecuencia respiratoria 19 r.p.m., presión arterial $120 / 70 \mathrm{mmHg}$, temperatura $36.5^{\circ} \mathrm{C}$, índice de masa corporal $29.4 \mathrm{~kg} / \mathrm{m}^{2}$, cabeza normal, piel y tegumentos con buena coloración e hidratación, cuello cilíndrico, sin adenomegalias palpables, tórax con mamas Tanner IV, abdomen globoso, distendido, peristalsis presente, no doloroso, sin visceromegalias ni tumoraciones palpables. Colposcopia sin datos relevantes. Resto de la exploración sin alteraciones.

Los exámenes preoperatorios (biometría hemática, tiempos de coagulación, química sanguínea y electrolitos séricos) fueron reportados dentro de los límites normales.

El perfil hormonal muestra: hormona estimulante del folículo $12.4 \mathrm{mUl} / \mathrm{ml}$, hormona luteinizante $4.01 \mathrm{mUl} / \mathrm{ml}$, progesterona $0.17 \mathrm{ng} / \mathrm{ml}$ y estradiol $34 \mathrm{pg} / \mathrm{ml}$.

Los marcadores tumorales indican: alfafetoproteína $4.2 \mathrm{ng} / \mathrm{ml}$, fracción beta de la gonadotropina coriónica humana $0.9 \mathrm{mUl} / \mathrm{ml}$, deshidrogenasa láctica $125 \mathrm{U} / \mathrm{l}$, antígeno Ca-125 45.13 U/ml, antígeno Ca-19.9 $553.17 \mathrm{U} / \mathrm{ml}$ y antígeno carcinoembrionario $3.3 \mathrm{ng} / \mathrm{ml}$.

En la evaluación con ultrasonido endovaginal de 5 a $10 \mathrm{MHz}$ Voluson ${ }^{\mathrm{TM}} \mathrm{E} 10 \mathrm{GE}^{\circledR}$ se encontró el útero de morfología y dimensiones normales, el ovario derecho con una tumoración de $3.98 \times 5.79 \mathrm{~cm}$ y el ovario izquierdo con una tumoración de $11.4 \times 10 \mathrm{~cm}$ (Fig. 1).

La paciente fue intervenida por laparotomía en mayo de 2019. En la exploración quirúrgica, el útero y las trompas de Falopio se encontraron íntegros. Se logró la enucleación de ambas tumoraciones y se preservó el tejido ovárico en su totalidad (Figs. 2 y 3).

Se enviaron ambas piezas para su estudio histopatológico, siendo la tumoración izquierda de $11 \times 10 \times$ $5 \mathrm{~cm}$ y la tumoración derecha de $6 \times 4 \times 4 \mathrm{~cm}$ (Fig. 4). Se confirmó el diagnóstico de tumor de células germinales benigno (teratoma quístico maduro bilateral) (Fig. 5). 


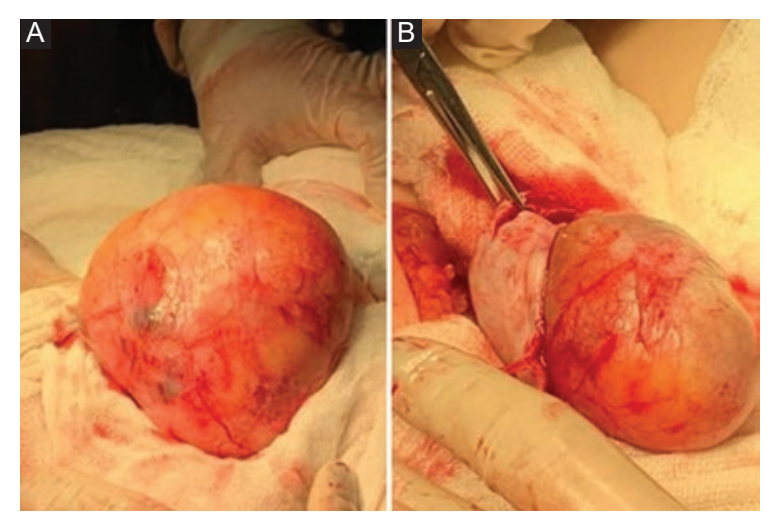

Figura 2. Visualización transoperatoria de ambos eratomas. A: teratoma izquierdo. B: teratoma derecho.

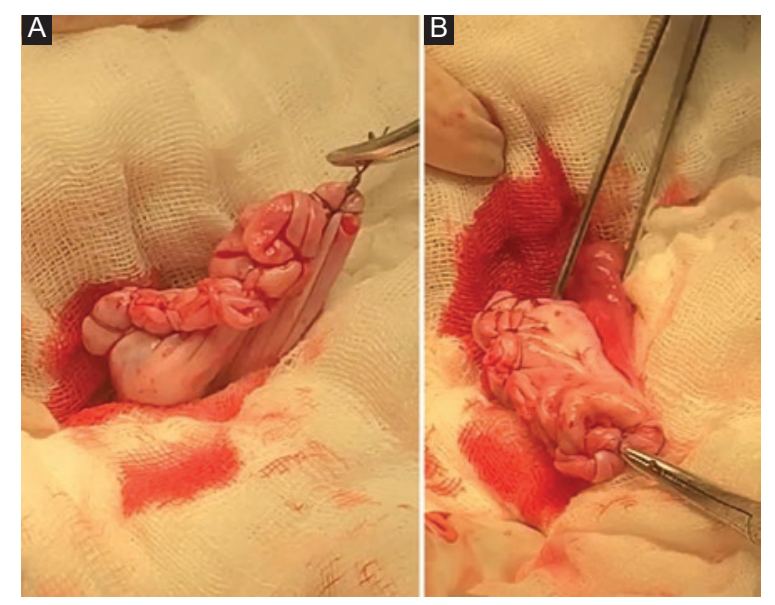

Figura 3. A-B: Imagen transoperatoria en la que se observa que se preservan íntegramente los ovarios.

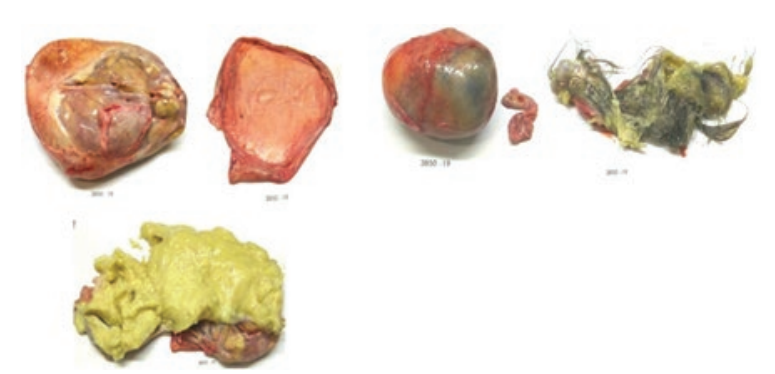

Figura 4. Tumores ováricos en disección por anatomía patológica.

La paciente fue dada de alta 24 horas después de la cirugía, tras un posquirúrgico sin ninguna anormalidad, y fue citada 7 días después, mostrando una evolución favorable.

Después de 30 días, la paciente volvió a su segunda cita de control, refiriéndose asintomática. Se le solicitaron nuevos estudios de control, principalmente perfil hormonal y un nuevo panel de marcadores tumorales, los cuales reportaron: alfafetoproteína $3.9 \mathrm{ng} / \mathrm{ml}$, antígeno carcinoembrionario $2.8 \mathrm{ng} / \mathrm{ml}$, fracción beta de la gonadotropina coriónica humana $0.05 \mathrm{mUl} / \mathrm{ml}$, deshidrogenasa láctica $110 \mathrm{U} / \mathrm{l}$, antígeno Ca-19.9 323.6 U/ml, antígeno Ca-125 23.2 U/ml, hormona luteinizante $5.16 \mathrm{mUI} / \mathrm{ml}$, hormona estimulante del folículo $8.10 \mathrm{mUl} / \mathrm{ml}$, estradiol $66.12 \mathrm{pg} / \mathrm{ml}$ y progesterona $1.04 \mathrm{pg} / \mathrm{ml}$.

Fue citada nuevamente en diciembre de 2019 para una tercera cita de control, en la que vuelve a manifestarse asintomática. Se le solicita un tercer perfil hormonal, en el que ningún parámetro estuvo fuera de rango y los marcadores tumorales permanecieron sin cambios significativos: alfafetoproteína $3.2 \mathrm{ng} / \mathrm{ml}$, antígeno carcinoembrionario $2.2 \mathrm{ng} / \mathrm{ml}$, fracción beta de la gonadotropina coriónica humana $0.0 \mathrm{mUl} / \mathrm{ml}$, deshidrogenasa láctica $111 \mathrm{U} / \mathrm{l}$, antígeno Ca-19.9 $20.5 \mathrm{U} / \mathrm{ml}$, antígeno Ca-125 $22.1 \mathrm{U} / \mathrm{ml}$, hormona luteinizante $4.1 \mathrm{mUl} / \mathrm{ml}$, hormona estimulante del folículo $8.6 \mathrm{mUl} / \mathrm{ml}$, estradiol $53.01 \mathrm{pg} / \mathrm{ml}$ y progesterona $0.99 \mathrm{pg} / \mathrm{ml}$.

Se le sugirió control anual mediante exploración física y ultrasonido pélvico, por el importante riesgo de recurrencia, además de tener cita abierta en caso de nueva sintomatología.

\section{Discusión}

Los tumores de células germinales, en contraste con otros tumores de ovario, se originan de células germinales primitivas que migran a la cresta gonadal hacia la sexta semana de vida del embrión, y por tanto este tipo de tumor puede exhibir un espectro de diferenciación histológica que imita el desarrollo primitivo embrionario ${ }^{5}$.

En la evaluación genética, las células que lo componen tienen un cariotipo normal $(46, X X)$, por lo que se considera que la diversidad de tejidos de su contenido no es producto de la fecundación; más bien se ha planteado el hecho de que su origen radica en el material genético propio del ovocito ${ }^{1}$. En estudios más recientes, Wang et al. ${ }^{6}$, mediante análisis de perfiles de ADN y estatus de metilación, plantean con sus resultados que el origen del teratoma maduro es más compatible con el concepto de la célula madre pluripotencial o de la célula germinal primitiva antes de la meiosis I.

Los tumores ováricos representan aproximadamente el $27 \%$ de las patologías oncoginecológicas, siendo la mayoría de ellos de origen epitelial (85-90\%). Los tumores no epiteliales abarcan el $10-15 \%$ restante, 


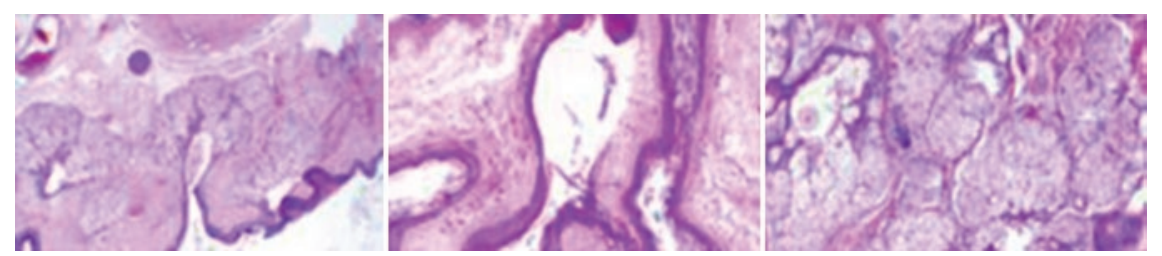

Figura 5. Histología del teratoma maduro benigno. Se observa la composición tumoral por diferentes tipos de epitelios maduros, epitelio cilíndrico, alternado con lesiones quísticas, cubiertos en la superficie por un epitelio plano estratificado con áreas de queratinización, folículos pilosos y glándulas sebáceas. En algunas zonas estas lesiones están cubiertas de células cúbicas, glándulas cubiertas de un epitelio cilíndrico, rodeadas de un estroma compacto y de tejido adiposo maduro.

Tabla 1. Clasificación histológica internacional de los tumores ováricos (Organización Mundial de la Salud) ${ }^{8}$

\begin{tabular}{lll}
\hline Tumores del estroma de los cordones sexuales & Tumores de células germinales & Tumores epiteliales \\
\hline - Células de la granulosa & - Disgerminoma & - Seroso \\
- Células de Sertoli-Leydig (androblastoma) & - Teratoma & - Mucinoso \\
- Tecoma & - Coriocarcinoma & - Endometrioide \\
- Fibroma & - Carcinoma embrionario & - Células claras \\
- De células esteroideas & - Del saco vitelino (seno endodérmico) & - Brenner (células transicionales) \\
& & - Epiteliales mixtos \\
& & - Indiferenciados \\
& - No clasificables \\
\hline
\end{tabular}

dividiéndose en dos grupos: tumores de células germinales y tumores del estroma de los cordones sexuales $^{7}$ (Tabla 1).

El cáncer epitelial de ovario por lo general ocurre en mujeres en la perimenopausia, mientras que los tumores de células germinales tienden a presentarse en mujeres jóvenes entre la segunda y la tercera décadas de la vida, etapa cumbre de su vida fértil, lo cual le confiere una complejidad extra cuando se considera que la gran mayoría de estas pacientes tendrá deseos de fertilidad, haciendo de esto otra prioridad dentro del abordaje y el tratamiento de esta clase de tumores ${ }^{8}$.

La presentación de las pacientes con tumores de células germinales ováricos resulta bastante similar en lesiones benignas y malignas, siendo el dolor abdominal (70-80\%) y la sensación de masa en el abdomen inferior los síntomas más frecuentes. La asociación de dolor abdominal y masa abdominopélvica palpable se encuentra en el $85 \%$ de los casos 9 . Esta clase de tumores suelen ser asintomáticos hasta que alcanzan grandes dimensiones, y más aún cuando llegan a comprimir estructuras vecinas ${ }^{9}$.

Para el diagnóstico, el abordaje adecuado es de vital importancia. Una anamnesis minuciosa, con particular énfasis en los antecedentes ginecoobstétricos de la paciente y en sus síntomas, es crucial en la evaluación. De igual forma, los antecedentes familiares no deben pasar desapercibidos. Los datos clínicos obtenidos del interrogatorio podrán ser de gran utilidad y orientar inicialmente el diagnóstico ${ }^{10}$.

En el abordaje de una masa anexial en estudio, la exploración deberá incluir palpación de las cadenas de ganglios linfáticos, auscultación pulmonar, palpación y auscultación abdominal.

El examen pélvico consistirá en inspeccionar visualmente el perineo, la vagina y el cérvix, además de complementar con tacto bimanual y examen recto-vaginal. Es importante mencionar que el examen pélvico puede tener bastantes limitantes, pero aun así se considera rentable, ya que existen hallazgos a la exploración que pueden orientar al médico a sospechar malignidad, como irregularidad de la masa, firmeza, nodularidad o presencia de ascitis ${ }^{11}$.

El ultrasonido transvaginal es el estudio inicial recomendado ante la sospecha o el hallazgo de una masa pélvica. Ningún otro método de imagen ha demostrado una superioridad sustancial que justifique su uso sistemático ${ }^{11}$. Las ventajas del ultrasonido transvaginal incluyen alta disponibilidad, buena tolerancia y molestias mínimas para la paciente, y costos accesibles. El ultrasonido Doppler permite un aumento en la especificidad diagnóstica, aunque siempre es recomendable ser cauteloso, ya que de cualquier forma no es posible tener certeza del tipo histológico del tumor por métodos de imagen ${ }^{12}$. 
Los datos que se consideran sugestivos de malignidad en la evaluación de una masa por ultrasonido son: quiste $>10 \mathrm{~cm}$, componentes sólidos 0 papilares, irregularidad, presencia de ascitis y Doppler color que demuestre un alto flujo. Las características ultrasonográficas compatibles con lesiones benignas incluyen: quiste pequeño, paredes lisas, ausencia de componentes sólidos, septos y Doppler que demuestra un bajo flujo sanguíneo ${ }^{13}$.

Los marcadores tumorales desempeñan un papel muy importante en el diagnóstico (principalmente diferencial) y el manejo del teratoma ovárico. Es necesario recalcar que, por definición, el teratoma maduro no tendrá producción hormonal de ninguna clase, pues por las características de su composición este evento resulta sumamente inusual, por lo que la negatividad en esta clase de parámetros jamás descartará la presencia de un teratoma ${ }^{14,15}$.

En lo que respecta al tratamiento, la única opción para los tumores de células germinales es la extirpación quirúrgica completa. Si no se logra obtener unos bordes quirúrgicos limpios en el estudio histopatológico, se debe plantear la reintervención. El procedimiento consistirá en ooforectomía en buena parte de las ocasiones, debido principalmente al tamaño del tumor o por no contar con la certeza diagnóstica de su tipo histológico. Solo en algunos casos selectos en los que el tumor en cuestión sea de proporciones menores, o se encuentre muy bien delimitado, podrá plantearse la enucleación de la lesión en búsqueda de preservar las funciones tanto hormonales como reproductivas de la paciente, en particular en casos excepcionales como la presentación bilateral del tumor $^{16}$, que representa una situación particularmente difícil, en especial en una mujer joven con deseos genésicos aún no cumplidos.

Algunos teratomas pueden tener componentes mixtos en su estructura; elementos inmaduros y malignos pueden coexistir aun sin haber elevación de los marcadores séricos. La mayoría de las veces no resulta posible contar con la certeza del tipo histológico del tumor, y por ello se recomienda una gran cautela en el abordaje quirúrgico y si surgieran nuevas dudas (principalmente a causa de las características del tumor) se recomienda también la realización de procedimientos de estadificación durante el transoperatorio ${ }^{17}$.

Dadas las características de esta clase de tumores y su predominancia en mujeres jóvenes, en edad reproductiva, será indispensable tener en cuenta sus deseos genésicos en todo momento, en especial en el planteamiento terapéutico y luego como parte integral de su manejo, siempre en aras de proporcionarle una mejor calidad de vida.

En caso de que el tratamiento de una tumoración ovárica implique una cirugía radical, existen varias opciones que se le pueden ofrecer a la paciente, según sea el caso (criopreservación de embriones, de ovocitos o de ovario) ${ }^{18,19}$.

La cirugía de conservación de ovario es posible gracias la existencia de un plano de disección entre los márgenes tumorales y el tejido ovárico sano ${ }^{20,21}$. En este caso, el tejido ovárico restante se evaluó macroscópicamente para detectar lesiones residuales, y el equipo médico que intervino a la paciente determinó la integridad y la viabilidad del tejido.

\section{Conclusión}

Existen algunos factores que pueden orientar al ginecólogo a plantear una ooforectomía en esta clase de casos, principalmente cuando se sospecha malignidad, si el tumor es grande o ante una torsión anexial, aunque siempre quedarán a criterio del médico tratante el abordaje y el plan terapéutico que se realizarán a la paciente.

Cuando el pronóstico de la paciente sea favorable (como en este caso), la preservación de la fertilidad y de la función gonadal deberá ser un objetivo prioritario en el tratamiento quirúrgico de los tumores de ovario. Es recomendable la cirugía conservadora como tratamiento de primera línea para todos los tumores ováricos, con excepción de aquellos que hayan sido catalogados como malignos y aquellos en los que la intervención quirúrgica urgente está indicada debido a la sospecha de torsión ovárica.

\section{Agradecimientos}

Los autores agradecen a la paciente de Hospital Santa Rosa de Lima por permitir, mediante el adecuado cumplimiento y derecho de privacidad, el estudio y la generación de este caso clínico.

\section{Financiamiento}

Los autores no recibieron financiamiento alguno.

\section{Conflicto de interés}

Los autores declaran no tener ningún conflicto de intereses. 


\section{Responsabilidades éticas}

Protección de personas y animales. Los autores declaran que para esta investigación no se han realizado experimentos en seres humanos ni en animales.

Confidencialidad de los datos. Los autores declaran que han seguido los protocolos de su centro de trabajo sobre la publicación de datos de pacientes.

Derecho a la privacidad y consentimiento informado. Los autores han obtenido el consentimiento informado de los pacientes y/o sujetos referidos en el artículo. Este documento obra en poder del autor de correspondencia.

\section{Bibliografía}

1. Linder D, McCaw BK, Hecht F. Parthenogenic origin of benign ovarian teratomas. N Engl J Med. 1975;292:63-6.

2. Wen-Chung W, Yen-Chein L. Genetic analysis results of mature cystic teratomas of the ovary in Taiwan disagree with the previous origin theory of this tumor. Hum Pathol. 2016;52:128-35.

3. Thomakos N, Malakasis A, Machairiotis N, Zarogoulidis P, Rodolakis A. Fertility sparing management in non-epithelial ovarian cancer. Which patients, what procedure and what outcome? J Cancer. 2018:9:4659-64

4. Steta-Mondragón JR. Teratoma bilateral y preservación de la fertilidad. An Med (Mex). 2012;57:325-31.

5. Talerman A. Germ cell tumors of the ovary. Curr Opin Obstet Gynecol. 1977;9:44-7.

6. Wang WC, Lee MS, Ko JL, Lai YC. Origin of uterine teratoma differs from that of ovarian teratoma: a case of uterine mature cystic teratoma. Int J Gynecol Pathol. 2011;30:544-8.
7. Serov SF, Scully RE, Sobin LJ. Histological typing of ovarian tumors. En: International Histological Classification of Tumors. Geneva: World Health Organization; 1973.

8. Pectasides D, Pectasides E, Kassanos D. Germ cell tumors of the ovary. Cancer Treat Rev. 2008:34:427-41.

9. Schneider DT, Terenziani M, Cecchetto G, Olson TA. Gonadal and extragonadal germ cell tumors, sex cord stromal and rare gonadal tumors. En: Schneider DT, Brecht IB, Olson TA, Ferrari A, editores. Rare tumors in children and adolescents. Berlin, Heidelberg: Springer; 2012. p. 327-402.

10. National Comprehensive Cancer Network. Genetic/familial high-risk assessment: breast and ovarian. Version 2.2016. NCCN Clinical Practice Guidelines in Oncology. Fort Washington (PA): NCCN; 2016.

11. American College of Obstetricians and Gynecologists' Committee on Practice Bulletins - Gynecology Gynecologists' Committee on Practice, Practice Bulletin No. 174: evaluation and management of adnexal masses. Obstet Gynecol. 2016;128:e210-26.

12. Harris RD, Javitt MC, Glanc $P$, Brown DL, Dubinsky T, Harisinghani MG, et al. Clinically suspected adnexal mass. ACR Appropriateness Criteria. American College of Radiology. Ultrasound Q. 2013;29:79-86.

13. Pavlik EJ, Ueland FR, Miller RW, Ubellacker JM, DeSimone CP, Elder J, et al. Frequency and disposition of ovarian abnormalities followed with serial transvaginal ultrasonography. Obstet Gynecol. 2013:122:210-7.

14. Cabezas-Palacios MN, Rodríguez-Zarco E, Rodríguez-Jiménez I, Márquez-Maraver F. Teratoma ovárico maduro e inmaduro, a propósito de un caso. Ginecol Obstet Mex. 2017;85:331-7.

15. Bridgewater JA, Rustin GJ. Management of non-epithelial ovarian tumours. Oncology. 1999;57:89-98.

16. Cecchetto G. Gonadal germ cell tumors in children and adolescents. J Indian Assoc Pediatr Surg. 2014:19:189-94.

17. Bailez MM. Laparoscopic ovary-sparing surgery in benign ovarian neoplasms. En: Bax KM, Georgeson KE, Rothenberg SS, Valla JS, Yeung CK, editores. Endoscopic surgery in infant and children. Berlin, Heidelberg: Springer-Verlag; 2008. p. 783-5.

18. Levine JM, Kelvin JF, Quinn GP, Gracia CR. Infertility in reproductive-age female cancer survivors. Cancer. 2015;121:1532-9.

19. Rienzi L, Cobo A, Paffoni A, Scarduelli C, Capalbo A, Vajta G, et al. Consistent and predictable delivery rates after oocyte vitrification: an observational longitudinal cohort multicentric study. Hum Reprod. 2012;27:1606-12.

20. Practice Committee of American Society for Reproductive Medicine. Ovarian tissue cryopreservations: a committee opinion. Fertil Steril. 2014:101:1237-43.

21. Oue T, Uehara S, Sasaki T, Nose S, Saka R, Yamanaka H, et al. Treatment and ovarian preservation in children with ovarian tumors. J Pediatr Surg. 2015;50:2116-8. 